\title{
Minimal Invasive Technologies for Treatment of HTS and Keloids: Medical Needling
}

Antigona Aliu and Matthias Aust

\section{Contents}

34.1 Background - 288

34.2 Introduction $-\mathbf{2 8 8}$

34.3 Method - 289

34.4 Effects of Medical Needling - 290

34.5 Needling Techniques -290

34.6 Postinterventional Treatment Measures - 291

34.7 Induction of the Post-Needling Wound-Healing Cascade - 292

34.8 Effects of Medical Needling Regarding Different Parameters - 293

34.9 Dermal Remodeling - 294

34.10 Improved Perfusion - 294

34.11 Dermal Thickness and Erythema - 295

34.12 Richness of Moisture - 296

34.13 Conclusion - 297

References -297 


\subsection{Background}

HTS (hypertrophic scars) and keloids remain a serious and challenging issue regarding medical and therapeutic intervention. Clinical symptoms such as pain or pruritus are followed by psychological deficits of stigmatization or even discrimination for the affected people. Considering the multifactorial pathogenesis of these pathological scars, the injury to the skin reaches the reticular dermis and causes subsequent aberrant wound healing. A secondary intention of wound healing is characterized by inflammation by promoting various tissue and immune cells. Hence, the vulnerable skin layer of HTS and keloids contains inflammatory cells, increased numbers of fibroblasts producing collagen and endothelial cells for a nutritive angiogenesis. This may promote chronic inflammation, which in turn may cause the invasive growth of keloids and explains clinical symptoms. The situation becomes even more complex with bacterial superinfection or other inflammatory responses, as proinflammatory cells and cytokines are highly upregulated. Pathophysiological parameters create a complex profile of HTS and keloids, which demand therapeutic attention and medical intervention.

\subsection{Introduction}

In this chapter, we are going to present medical needling as an ideal therapy for the treatment of hypertrophic scars and keloids. The past has shown difficulties in treating these types of scars that are characterized by a complex anatomy and a progressive degradation of the scar texture. Serious challenges for various therapy approaches are a rapid and uncontrolled growth, a lack of moisture and a rigid structure. Affected people are confronted with dysfunctional and aesthetic deficits in their daily life or suffer from further harm through stigmatization. For this indication, medical treatment is required that replaces surgical interventions and other ablative treatments. HTS and keloids are prominent scars that frequently occur in combination with persistent erythema. Widespread and deep damage of the skin layers is often followed by secondary wound healing, which enhances the formation of either HTS or keloids. Keloid scars occur when the skin overreacts to the injury, after which they continue to grow and get dark in color. The histopathology of keloid demonstrates an extensive tissue proliferation beyond the margin of primary wound. Dark skin types are predisposed for the development of keloids that appear when a forced wound closure proceeds under tension. That is why keloids can cause great discomfort, tightness, or a limited range of mobility if they develop near a joint such as the knee or ankle. An excessive stretching of skin creates uncomfortable pres- sure over the fibrotic tissue, which can cause itching. Because of their typical larger size, they tend to rub on clothing, causing unbearable irritation.

Whereas the development of HTS is fairly manageable, keloids reveal specific features concerning the parameters of size and growth. Keloid scars develop uncontrolled or excessive fibrogenesis and are a tremendous source of collagen that still causes clinical problems until now. Thus, keloid is an active tissue that demonstrates signs of inflammation such as redness, itch, and mild pain. The permanent tendency to uncontrolled growth exceeds the original wound area and reaches massive proportions due to uncontrolled fibrosis. The last-mentioned issue is associated with a dysregulation of growth factors, which leads to an overproduction of scar tissue and an uncontrolled synthesis of the extracellular matrix. Hence, therapeutic treatment of keloids differs from those of normal hypertrophic scars. Keloid is often recurrent, although it has been treated with either pharmacological agents or surgery. Dermatological approaches of scar removal are not based on ablative treatment methods. They include compression therapy, intralesional corticosteroid injections, and excisions. When injected into the keloid, appropriate medication helps shrink the scar for a short time. The patient is due to receive a series of injections once every month, which means a constant therapy in order to prevent renewed scarring. Moreover, the use of radiation therapy instead of laser therapy is a common practice. However, this method also means a radiation exposure, which is always a health hazard. Ionizing radiation should only be implemented when medical indication is given or other alternatives are not reachable.

With regard to HTS, ablative treatments such as surgical interventions show temporary success by reducing the scar tissue to the level of the surrounding skin. These circumstances support the risk of recurrence and a continuous degradation of scar texture and healthy skin. The majority of conventional treatments are based on ablative and radical principles that provoke short-term responses and only suited for the removal of HTS. For this reason, conventional therapy approaches show little success and permit poor access to an efficient treatment of keloids or HTS.

Against this background, the demand for less invasive and effective functional and aesthetic treatments is steadily growing and remains a permanent issue in modern medicine. The focus is clearly defined by the patient's satisfaction with the aim of maximal outcome and minimal risk at the same time. Medical needling seems to be an ideal method for what is postulated in many fields of modern medicine. PCI displays a simple regenerative technique that affects skin-related indications and in particular scars. Its success is manifested in skin regeneration particularly for severe and wide- 
spread scars with hypertrophic and keloid features. The method of PCI requires neither expensive apparatus nor the need of complex instruments and sets a new trend in plastic and aesthetic medicine. By stimulating complex signal transduction pathways in the postneedling wound-healing cascade, the natural regeneration process is modified and, hence, is more efficient. According to that, medical needling improves the appearance and quality of scars with comparatively low risk and stress for the patient. The postneedling cascade induces gene expression and the proliferation of skin and stem cells that are important for dermal remodeling. The expression of specific proteins and reorganization of the extracellular matrix affect epidermal thickness and creates a stable and functioning skin barrier [1]. Furthermore, the PCI-induced synthesis of collagen and release of endogenous growth factors allow an association with scarless wound healing. Epidermal as well as dermal structures remain intact and histological findings show a complete re-epithelialization of the epidermis after treatment.

Current medical standards offer a diversity of noninvasive treatments (e.g., silicone patches) and minimal invasive procedures (e.g., cortisone injections) or surgical options such as scar excisions, tissue transfer, and W- and Z-plasties. Keloids show a limited therapy spectrum, which is mainly based on nonablative treatments (radiation, injections). Ablative methods such as laser resurfacing, dermabrasion, and deep chemical peels are all together defined by the same principle and find use in the treatment of HTS: they lighten the scar by destroying the skin structure and provoke an inflammatory response. As a result, the treated area is replaced by a thinner epidermis that shows flattened rete ridges and parallelly orientated scar collagen that is typical for fibrosis [2]. Furthermore, the skin is more vulnerable for bacterial or viral infections and external stress. These complications make a successful treatment of hypertrophic and keloid scars almost impossible, as they already have dysfunctional qualities. Complex structural and physical features of keloids have shown that common methods display temporary solutions that conceal the actual problem instead of improving the scar quality. In most instances, the scar quality of keloids or HTS would be much worse then. Compared to that, PCI has been proved to be a relatively simple, fast, and controlled method, which can be safely repeated and used in body regions where ablative or semi-ablative treatments have limited usage. Current studies on medical needling show a positive effect on hypertrophic scars regarding the parameters elasticity, moisture, transepidermal water loss, and erythema. In terms of dermal reorganization, high levels of structural proteins - glycosaminoglycans and proteoglycans - as well as an increased presence of physiological collagen are measurable after needling. PCI preserves dermal structures and promotes the formation of physiological collagen instead of scar collagen. For this reason, PCI overcomes shortcomings of other conventional methods that are available for treating problematic scars such as HTS or keloids. Study outcome has already shown the positive effect of medical needling on HTS in the past years and latest clinical data reveal similar results concerning keloids.

\subsection{Method}

Medical needling is based on a simple idea of puncturing the affected area repeatedly with a roller covered with needles of a specific length. Approximately 20 years ago, Camirand and Doucet had the experience that treating hypertrophic scars with a tattoo gun affects the scar texture and achieves significant improvements regarding clinical parameters [3]. Based on these ideas, Fernandes developed the technique of percutaneous collagen induction. In 1997, he realized this scientific discovery by establishing a roller equipped with needles in order to produce thousands of neighboring micro-wounds in the dermis, which cause intradermal bleeding. Thanks to targeted research within the last 15 years, scientific data have been provided by Aust et al. at first, and underline the efficacy as well as safety of medical needling.

In Germany, medical needling is now a licensed therapy for the treatment of scars and other related indications. The needling device is covered with needles of the desired length $(0.5-3 \mathrm{~mm})$ and needs to be rolled over the scar in three directions under constant pressure: vertically, horizontally, and diagonally. In the case of hypertrophic scars and keloids, surgical needling with a needle length of $3 \mathrm{~mm}$ is instructed in order to reach the scar collagen as deeply as possible. A straight guidance of the device is necessary in order to prevent shear forces and deeper damage. Relative to the extent of the scar size, this procedure requires 30-60 minutes of mechanical exposure. The penetration of the papillary dermis leads to thousands of micro-wounds and intradermal bleeding through the parenchymal canals. Minimal lesions of the epidermis do not impair basal layers containing stem cells with regenerative capacity. The increased expression of specific growth factors and release of structure proteins induce a modified wound-healing cascade with a great regenerative potential. The scar is sufficiently needled when multiple and confluent ecchymosis as well as skin swelling are clearly indicated. After 24 hours, epithelial cells close the channels and are reorganized into a natural protection barrier, which reduces the risk of potential postoperative complications such as infections. Therapy benefits are optimized by the application of nourishing products in first 24 hours. Swelling and local redness or edema of the treated area disappears after approximately 4-7 days. PCI is performed under general 


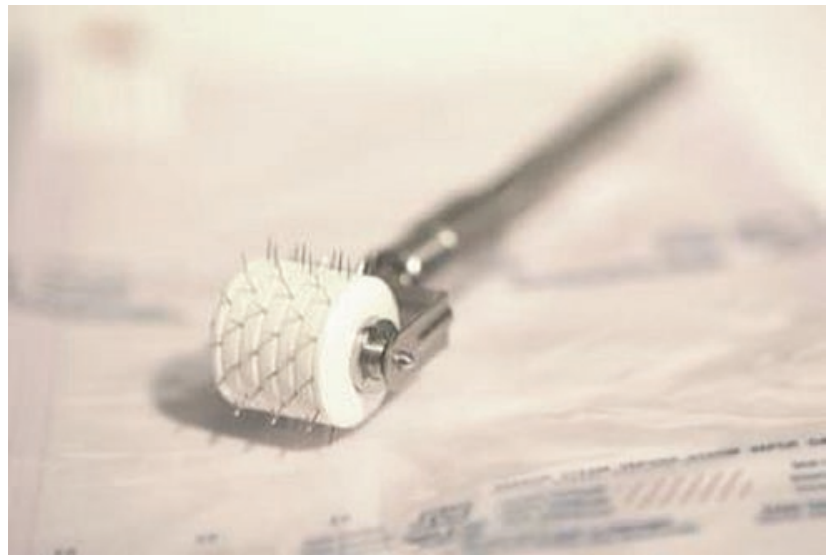

- Fig. 34.1 Roller device for medical needling

anesthesia in an operation theatre. When smaller areas are treated, the performance can be done under local anesthesia. Preoperative formalities include an informed consent form signed by informed patients or parents of patients younger than 18 years of age. Pre- and postoperative management is kept simple and includes the application of Vitamins A, C, and E as antioxidants [4]. According to latest research, maximal therapeutic outcome regarding epidermal thickness and a better prognosis for rapid healing can be expected. Postoperative monitoring is not substantially necessary. However, after surgical needling, a short down time of a few days needs to be considered, as the procedure requires general or local anesthesia. Therefore, a stay in hospital is only recommended, not obligated. Nevertheless, patients are able to get back to their daily life after a short time of recovery (• Fig. 34.1).

\subsection{Effects of Medical Needling}

The intradermal bleeding caused by the mechanical procedure induces second-messenger substances of significant signal transduction pathways, which lead to a modified physiological regeneration of the skin. Specific features of both skin layers are not only preserved in their functional structure but also promoted in a positive qualitative way. Epidermis and dermis remain functionally intact and protect subcutaneous structures. Moreover, single layers of the epidermis become thicker, which guarantees great stability and a reduced vulnerability. Treated skin reacts less sensitively to damaging physical factors such as UV radiation that is associated with postinflammatory hyperpigmentation [5]. Furthermore, medical needling is also suited for all skin types. Treating dark skin is not followed by any dyspigmentation or even hypopigmentation, although dark skin is indeed predisposed for color shifts due to the naturally increased amount of melanin. Molecular and cellular processes induced by PCI affect neither the amount of melanocytes nor the mechanisms of synthesizing melanin. However, PCI specifically modifies the expression levels of the melanocyte-stimulating hormone (MSH) and Interleukin-10 (Il-10). MSH influences the activity of melanocytes and is downregulated within the postneedling cascade. On the other hand, high levels of Il-10 are evident, which is an antiinflammatory cytokine, and support immunological tolerance. As a targeted therapy, PCI concentrates especially on those areas that are aimed to be treated and also affects the surrounding healthy skin in a positive way. Due to the fact that needling does not create an open wound surface, there is a low risk for the development of viral or fungal infections, which could complicate a scar-free wound healing.

\subsection{Needling Techniques}

The needling treatment needs to be planned and prepared in advance. This includes the examination and categorization of the scars according to specific features. The selection of the appropriate length of needles depends on the type of scar, pain management, and expectation from the patient's side. From this perspective, downtime repetition capacities and aesthetic achievements are considered. Massive scars with intense deficits would be HTS and keloids that are predominantly treated by surgical needling. This means a longer downtime but also maximum results. Considering input and outcome, maximizing the patient's expected results is aimed to achieve.

In principle, medical needling can be classified into cosmetic, medical, and surgical needling. The difference depends on the length of the needles used and the intensity of the physiological effects as well as the postoperative regime they require. First mentioned types of needling play a minor role for the treatment of HTS or keloids, as the desired effects cannot be achieved.

Cosmetic needling is performed as a purely cosmetic treatment with needles $0.1-0.5 \mathrm{~mm}$ in length for structural and superficial skin modifications. The needling procedure does not cause any intradermal bleeding and hence any postinterventional reactions remain unnoticed. Using the minimal length of needles does not require any anesthesia and allows repeating the treatment daily. The patient does not experience any downtime and gets back to his daily routine without any complications. All together, the cosmetic needling is used as a penetration enhancer for topical skin care products.

Medical needling can be defined as PCI when the needles have a length between 1 and $2 \mathrm{~mm}$. Needles of this length reach just beyond the basal membrane and lead to minimal petechial hemorrhages in the papillary dermis, which activate the TGF- $\beta$ signal cascade and give a skin- 
regenerating effect. The needle length is proportional to the provoked bleeding, as it gets more intense when longer needles are used. However, intradermal lesions are so small that there is a minimal downtime without obvious edemas or bruises. Moreover, effective topical anesthetics can be used to make the process nearly painless. Afterward, the treated area might be reddened and needs a few days to recover and normalize.

From the perspective of clinical efficacy, surgical needling proves to be more intense and impressive when the performance is done with a needle length of $3 \mathrm{~mm}$. That is why HTS and keloids are indications for surgical needling. This procedure is carried out under general or local anesthesia and can also require a stay in hospital. The puncture depth does not only affect the epidermis and dermis but also the upper layers of the subcutis including the vascular system that is responsible for the desired and controlled bleeding after needling. This mechanical prodecure affects the skin structure and function by provoking stress and regenerative resources at the same time which is aimed to improve the natural skin barrier with intact skin layers. For this reason this step needs to be performed wisely and carefully observed. The relatively painful procedure under local anesthesia provokes primary inflammatory woundhealing reactions with temporary erythema, swelling, and bruising. The needling device needs to be rolled over the affected area until these signs of sufficient needling are apparent. The potential for aesthetic correction and reduction of the scar is on the highest level and provides maximal outcome. When selecting a needle length of $3 \mathrm{~mm}$, the patient is expected to accept a protracted downtime in order to achieve maximal results in the phases of recovery. According to that, longer recovery periods of weeks should be taken into account (• Figs. 34.2 and 34.3).

The selection of the needle length varies relative to the indication and its intensity of cicatrization. Moreover, there are important differences notable: The

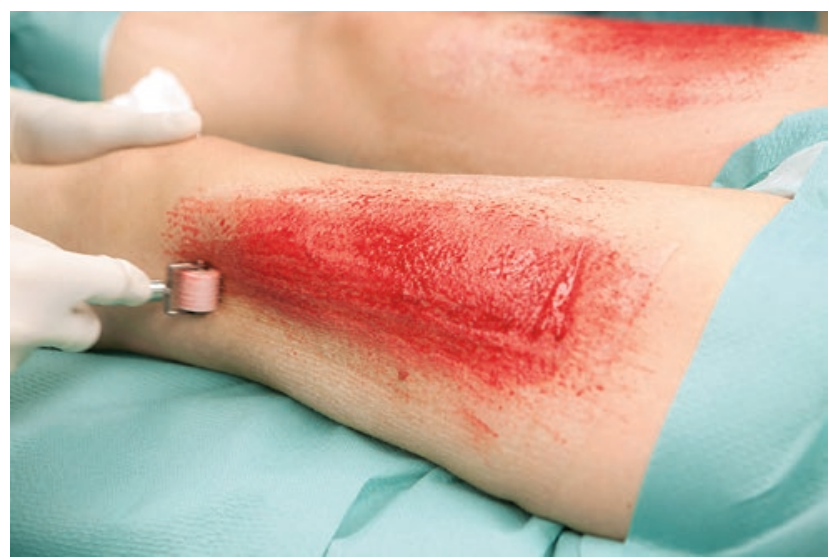

- Fig. 34.2 Technique of surgical needling performed with a needle length of $3 \mathrm{~mm}$. Desired bleeding and marked edema induce therapeutically effective collagen production during the postneedling wound-healing cascade

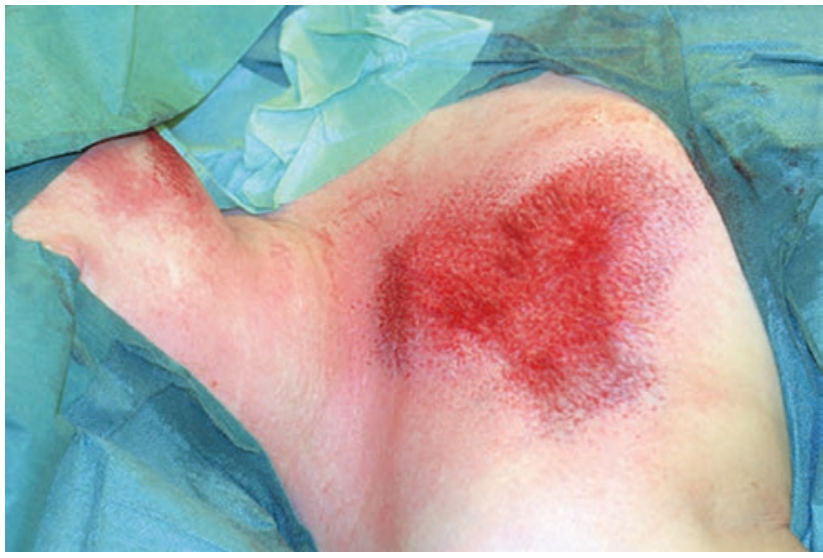

Fig. 34.3 A heavier bleeding caused by the $3 \mathrm{~mm}$ needling method

shorter the needles, the more often treatments need to be carried out in order to reach desired effects. Longer needles are indicated when treating prominent kind of scars such as HTS and keloids. Summing up, the combination of the patient's expectations and the desired result decides the amount of therapies and the selection of the three possible needle lengths.

\subsection{Postinterventional Treatment Measures}

Postoperative Wound Management - For optimizing and maximizing therapy outcome, it is instructed to treat the skin sufficiently and effectively under pre- and postoperative circumstances. The application of vitamin A and antioxidants such as vitamin $\mathrm{E}$ and $\mathrm{C}$ is necessary to achieve best results. A specific skincare that combines the above-mentioned vitamins with a strongly recommended gentle cleansing washing lotion containing tea tree oil should be performed every 2 hours as long as the needling canals are still open. While the skin remains bruised and swollen, direct exposure to sunlight should also be avoided. A moist wound-healing regime of vitamin-based creams prevents crust formation after the initial bleeding, which could eventually impair the final results. The loss of serous fluid through the puncture holes in the immediate postinterventional period can lead to crusting, which needs to be carefully averted by washing off any surface serum. In consequence, preventing crusting reduces the risk of bacterial superinfections. Moreover, inflammation and secondary wound healing associated with the development of HTS or keloids can be avoided.

Managing Complications - As needling does not produce open wounds, the postoperative complication rate is very low and postinterventional monitoring is not necessarily needed. However, local edema 
can develop with longer needles, especially done on large areas. It is recommended to keep the patient in the clinic for a few hours after treatment. It needs to be considered that after extensive surgical needling under general anesthesia, the patient may experience burning pain in the treated area. Therefore, pain should be managed with effective drugs particularly in the first hours postoperatively. The use of nonsteroidal antiphlogistic medication should be avoided, as the primary inflammatory response is desired. However, keloids and HTS require regular follow-up examinations in order observe wound-healing processes and to ensure that renewed scarring or proliferating tissue can be excluded. In the case of keloids, a further extension of the scarring is not that rare after treatment. Clinical data also reveal the formation of necrotic tissue when there is an inadequate blood supply. Special attention needs to be paid to patients with diabetes that shows difficulties in wound healing anyway.

The intradermal bleeding caused by PCI is the essential driving force in medical needling. In this context, the endogenous potential of natural wound healing induced by PCI differs from the conventional inflammatory response to traumatic incidences starting minutes after injury. Therefore, the difference between the postneedling regenerative cascade and the conventional woundhealing cascade becomes very clear. The PCI-induced regenerative process is based on completely different mechanisms that are associated with the desired bleeding, as plenty of blood vessels are pierced at the same time. However, an open wound surface is not created within this procedure that would need to be refilled by regenerative fibers in the course of conventional wound healing. Refilling can rapidly escalate into an overproduction of tissue fibers, which again supports the development of HTS and keloids. The extent of the initial bleeding and the excretion of serous fluids can appear in different intensities and mainly depend on the area treated as well as the needle length that has been specifically selected. In consequence, facial skin regions show a higher bleeding capacity, as they tend to react more sensitively to mechanical stress. Further, these regions are more affected by swelling and bruising because skin seems to be thinner and therefore reacts more intensely to operative interventions than the skin of other regions. Eventually, PCI-related results are achieved by replacing scar typical collagen with normal collagen of healthy skin [6].

\subsection{Induction of the Post-Needling Wound-Healing Cascade}

The intended trauma through the repetitive puncturing initiates a physiological wound-healing cascade. Platelets and neutrophils secrete growth factors such as the platelet-derived growth factor (PDGF), fibroblast growth factor (FGF), vascular endothelial growth factor (VEGF), tissue growth factor, and transforming growth factor- $\alpha$ and $-\beta$ (TGF- $\alpha$, TGF- $\beta$ ). The interactive cooperation of these factors provides blood coagulation, as well as the synthesis of dermal structures such as collagen, elastin, and fibronectin. Furthermore, the differentiation and migration of fibroblasts and keratinocytes contribute to regenerative processes. Fibroblasts have essential roles in synthesis of collagens I and III. Other cells that are represented in all tissues including the dermal layer are fibrocytes, dendrocytes, mast cells, and further immune cells secreting collagen I and collagen III protein. Seen from a dermatological view, TGF- $\beta$ has specific functions that have a key role in the postneedling wound-healing cascade. In general, wound healing can be divided into three phases interacting through numerous growth factors and other essential elements, into approximately month-long sequences: inflammation, proliferation, and regeneration (• Fig. 34.4).
- Fig. 34.4 Wound-healing course schematic representation

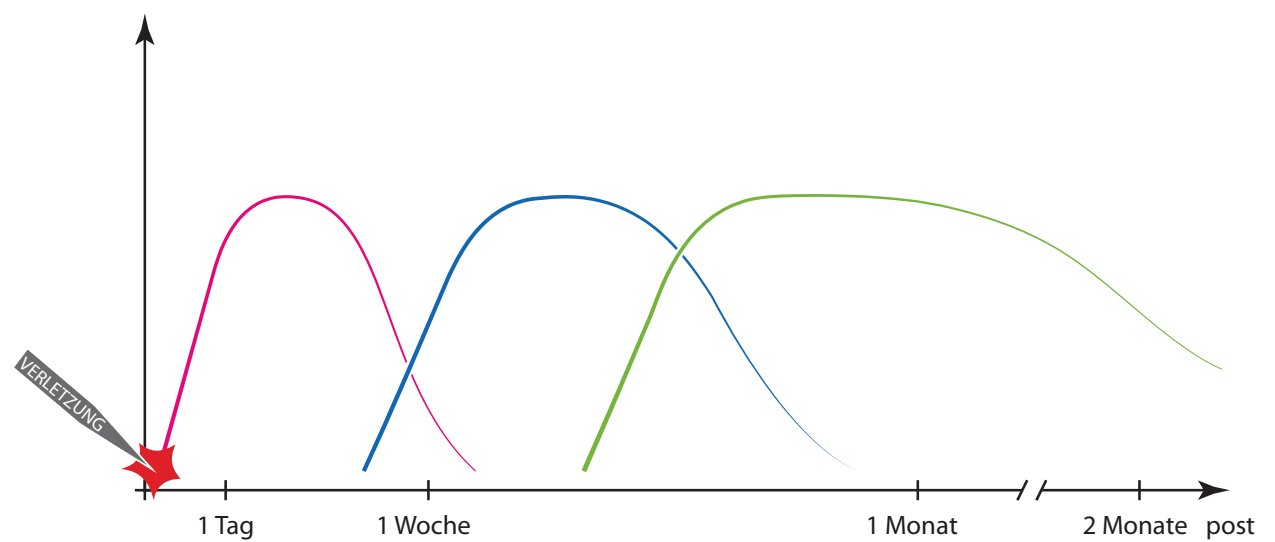

Inflammatory phase

Proliferation and Epithelialisation
Reparation and Regeneration 
Right after the injury, the natural and typical reaction of inflammation starts by activating immune cells, blood coagulation, as well as vasoconstriction [7]. The following stage of proliferation is marked by growth factors, especially focused on TGF- $\beta$, which is important for the regulation of growth processes. Dysregulated signal transduction pathways can lead to a malignant degeneration of cells, which can result in the development of cancer or malignant tumor in the worst case. Keloids have indeed characteristics that are similar to tumor cells. The persistent presence of TGF- $\beta 1$ and- $\beta 2$, as well as the absence of TGF- $B 3$ in the conventional wound-healing process, indicates the formation of typical rigid scar tissue. The activity of fibroblasts is necessary for the regeneration of the extracellular matrix and the synthesis of collagen. After the phase of regeneration, an intact skin barrier of renewed epithelial cells and a mechanically stable skin structure are evident. These are single steps described in a typical wound-healing procedure. On the contrary, the PCI-induced wound-healing mechanism is based on a modified principle of what is already known and described regarding wound healing. Special focus is manifested in the modified TGF- $\beta$ signal transduction pathway. Whereas TGF- $\beta 1$ and 2 are significantly downregulated in the postneedling cascade, TGF- $B 3$ shows a dominant activity 24 hours postinterventionally. Against this background, the traditional wound-healing paradigm is converted into a regenerative phase marked by the synthesis of structural collagen of healthy and vital skin instead of scar tissue. This is why there is a low risk for the development of hypertrophic or keloid scars where it usually comes to an uncontrolled hyperplasia of collagen fibers and an uncontrolled growth of the connective tissue. Very common consequences are rigid and difficult scars.

\subsection{Effects of Medical Needling Regarding Different Parameters}

Scarless Wound Healing - In order to avoid secondary wound healing, a controlled activity of growth factors is necessary. The close relation between TGF and the formation of collagen type I fibers represents a key role in repair and regeneration mechanisms. In this context, it needs to be considered that wounds healed by secondary intent tend toward an uncontrolled proliferation, which enhances the development of HTS or keloids. Type I collagen is the physiological desired collagen of lattice pattern structure in healthy and vital skin, whereas collagens type III shows parallelly orientated fibers that is typical for scars. TGF- 31 and 2 are at their highest levels of activity when a dense collagen structure of type III in scars is built. Study results have proved that in keloids with excessive cell proliferation, the expression of procollagen I and III -mRNA as well as -protein is increased. In excessive cell proliferation of keloid tissue, there is a dominant presence of collagen III, although both collagen I and collagen III seem to be increased. Significant differences to normal skin are manifested in the shift of the collagen I and III ratio toward collagen III. However, a significant downregulation of TGF-B1 and 2 is evident in scar-free, embryonal wound healing determined by TGF-B3. Second kind of wound healing is still active in juvenile tissue to a lesser extent, but not present in adult tissue that is rather predisposed for the development of scars. High levels of TGF-B3 in the postneedling cascade do not only induce the synthesis of collagen type I, but also manage the conversion from collagen type III to the desired type I collagen (• Fig. 34.5).
Fig. 34.5 Regulation of transforming and growth factor, TGF-B3, and scarless healing. Microarray analyses of TGF- $B 1,-\beta 2$, and $-B 3$ expression levels in treated and untreated animals show that the needling treatment stimulates TGF- $B 3$ to a greater extent than TGF- $\beta 1$ or $-\beta 2$. Moreover, the induction of TGF- $B 3$ gene expression continues even beyond the initial wound-healing phase, whereas the two other genes are downregulated during the second week postneedling

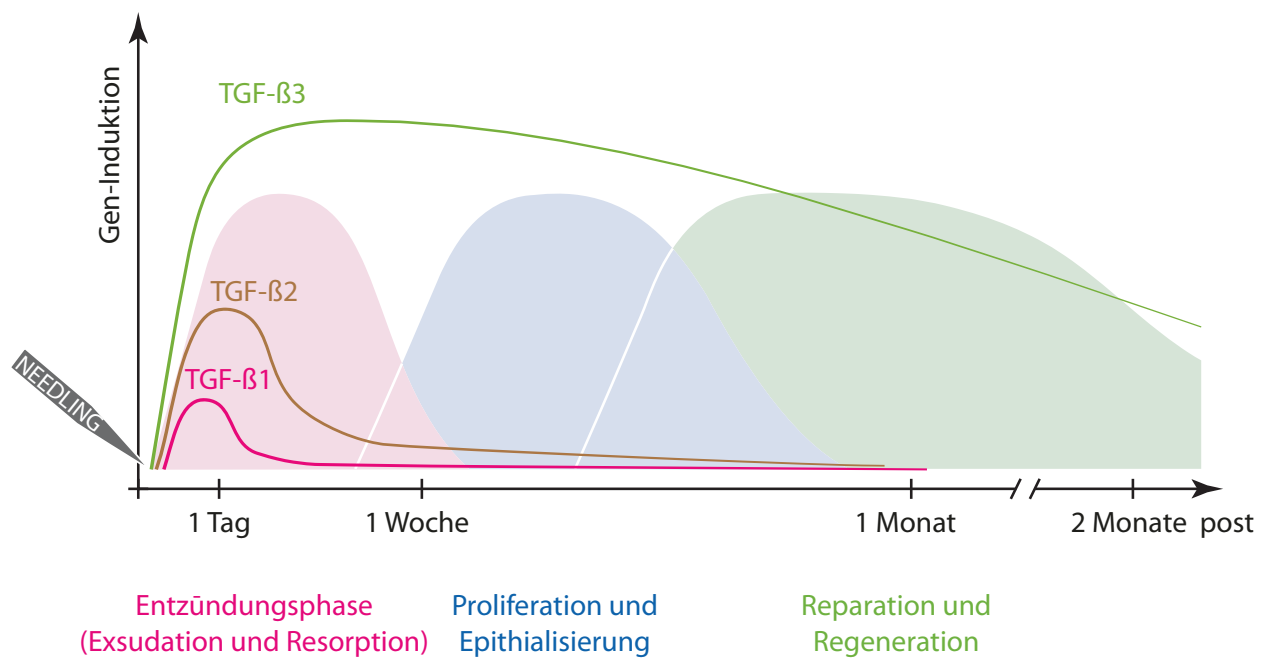




\subsection{Dermal Remodeling}

Furthermore, PCI modulates the expression of relevant genes and molecules that are responsible for remodeling the extracellular matrix. The skin appears thicker, more vital, and rejuvenated. Therefore, PCI displays a method that is focused on both quantitative and qualitative improvement of dermal function and appearance. The release of structural proteins, including fibronectin and glycosaminoglycan molecules, as well as the activity of specific enzymes, achieves dermal remodeling. These components of the postneedling cascade stimulate the proliferation of keratinocytes and other skin cells in the basal layer of the epidermis, which changes the physical and biological characteristics of skin. The entire connective tissue framework seems to be thicker and denser. Other endogenous factors contribute toward improved skin elasticity in terms of dermal remodeling, as the amount of elastin is significantly greater after medical needling. A lack of elasticity is typical for HTS and keloids and one reason for their rigid structure, which puts the scar as well as the surrounding healthy skin under a great pressure. Hence, flexibility and mobility of the affected area are compromised, which might be a problem when regions close to joints are affected. Not only for this reason, TGF- 33 reaches high levels of expression in the initial phases but shows a declining trend in the following phases of proliferation and regeneration. Based on Furguson's work, the initial dominant influence of TGF-B3 is important for the PCI-related wound-healing mechanisms. However, clinical expertise shows that giving TGF-B3 additionally in phases of normally low levels can eventually impair regenerative processes. In conclusion, the method of medical needling influences the release and immediate effect of TGF, as well as the synthesis of collagen type I interacting within the process of dermal reorganization and remodeling. Once again, the signal transduction pathway of TGF seems to be one of the most important PCI-related factors. Therefore, the method of PCI has a regulatory function of promoting a controlled activity of growth factors and other regenerative factors as well as cytokines. With regard on HTS and keloids, the main focus is on managing growth and hypertrophy by a controlled activity of growth, epithelial and vascular factors (• Figs. 34.6 and 34.7).

\subsection{Improved Perfusion}

Another factor showing high concentration during the postneedling regenerative cascade is VEGF. In terms of angiogenesis, its main function of creating new blood vessels and enhancing perfusion accelerates wound-

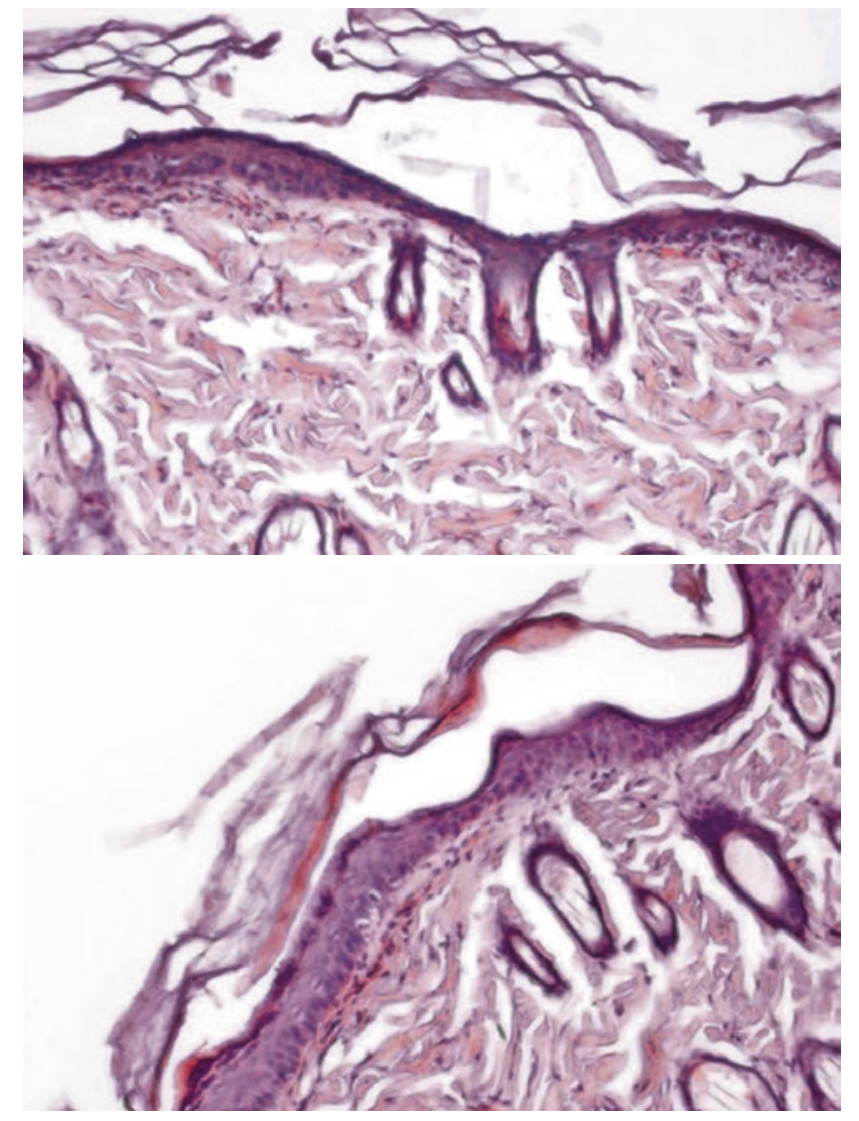

- Figs.34.6 and 34.7 Regenerative effects on the epidermis through needling. One day after the treatment, Masson's trichrome staining shows no injuries in either the epidermis or the dermis. Both skin layers are functionally intact; there are no signs of ablation or cell damage. • Figure 34.6 Untreated animal (control). • Figure 34.7 24 hours postneedling

healing processes. The method of PCI provides a controlled and limited release of VEGF in order to prevent dysregulated activity, which can lead to degenerative processes. High levels of VEGF are initially observed in the postneedling cascade, which are again not kept constantly high at all times. Hence, medical needling has a positive effect on subcutaneous structures conveyed by VEGF as an important indicator for active angiogenesis. The expression of this growth factor induces the vascular system by supporting the proliferation and activity of vascular endothelial cells [8]. This process happens after the initial inflammatory response of a vasoconstriction with the primary intention of stopping the bleeding as a typical reaction to an injury. The restricted perfusion is then followed by a massive vasodilatation in the wound-healing phase. The regulative effect on the vascular system explains the intense bleeding after the needling procedure, which is in fact an enhancer of the wound-healing progress. The better the blood perfusion of the skin tissue, the greater the bleeding after mechani- 
cal stress induced by PCI, which accelerates healing processes. The limited activity of VEGF for a desired period of time (postinflammation) has the advantage of a rapid wound healing due to a better perfusion and a primary wound closure in a timely and effective way. For this reason, the risk of secondary wound healing can be excluded, as delayed wound-healing processes are very improbable and also not expected after needling. In this context, the development of HTS and keloids is once again kept at minimal risk.

\subsection{Dermal Thickness and Erythema}

An effective wound-healing process implies active vascularization and causes a temporary reddening of the skin, which is not associated with persistent erythema. After needling, the affected area is swollen and reddened due to a better perfusion in the course of effective healing processes. However, the problem of striking discolorations of especially hypertrophic and keloid scars remains a serious one for the affected people, as the scar color differs extensively from the surrounding healthy skin. Discolorations can reach from slightly reddened skin or persistent erythema to dyspigmentation or even hypopigmentation, which is quite typical for HTS. Untreated hypertrophic scars tend to a thin and vulnerable epidermis that is unstable and more transparent for local perfusion. This might support the appearance of erythema through a thin skin texture with a long-term impact. After needling, the intense blood circulation is less prominent and temporarily limited through a thickened epidermis. The entire tissue structure is more compact and makes the great optical difference between scar and surrounding skin less prominent. Either way, medical needling represents a skin-normalizing therapy method and creates a homogenous image of initially reddened scars toward healthy skin. Based on outcomes of objective measurement methods with the Mexameter, medical needling achieves a normalization of the skin color and an almost complete adjustment of the scar tissue toward healthy skin after repetitive treatments. As the method is based on percutaneous collagen induction, the synthesis of collagen improves vital thickness, which is directly associated with less transparency and a low risk for the development of erythematous hypertrophic scars (• Fig. 34.8).
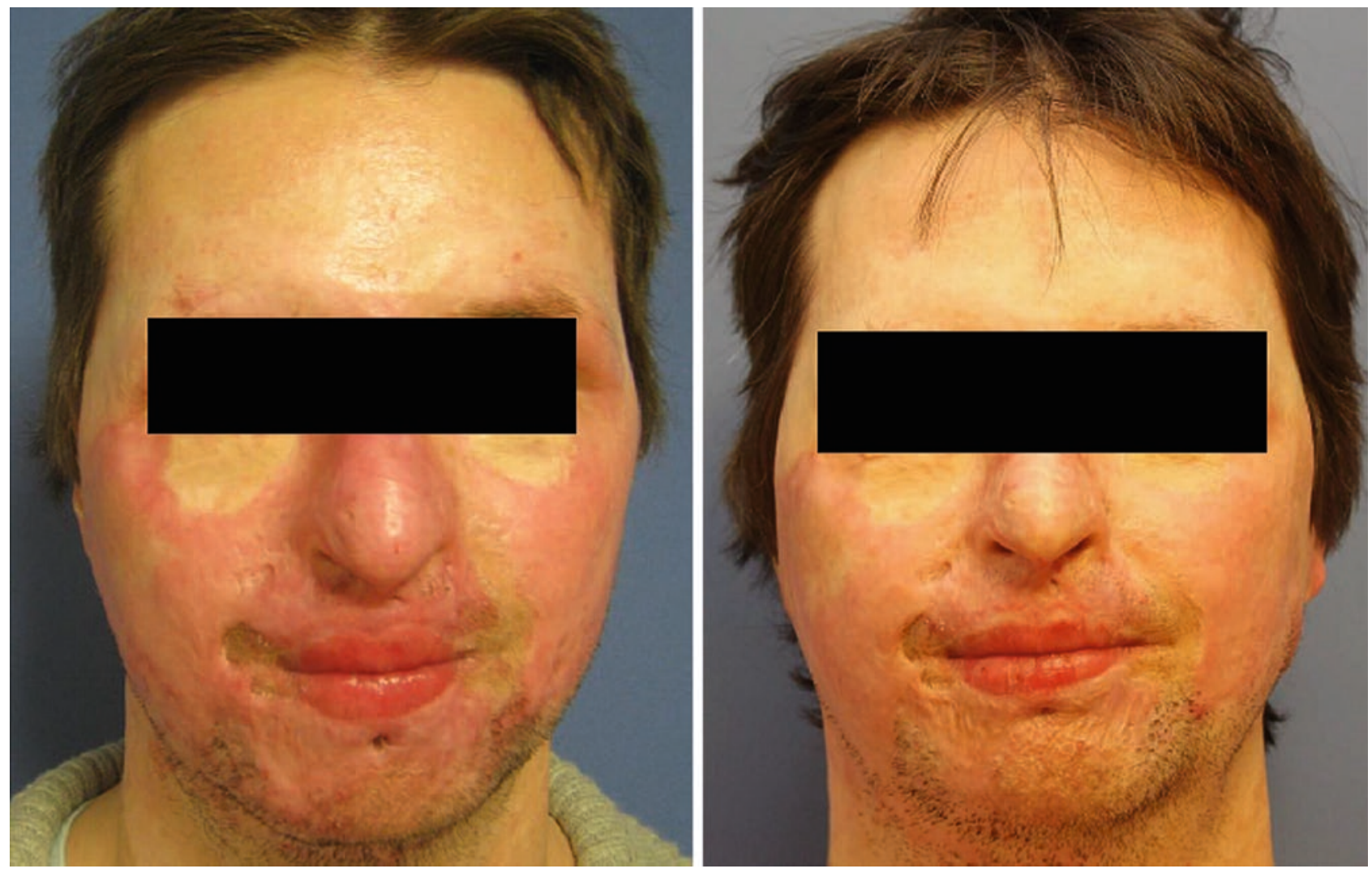

- Fig. 34.8 Patient 1, frontal shot, preoperatively (left) and one year postoperatively after needling (right). Areas treated include the entire face. Reduced erythema and hypertrophy of the fibrosis 


\subsection{Richness of Moisture}

HTS and keloids are especially characterized by a lack moisture, which combines typical visible anomalies with symptoms of pain and itching. The current difficulty of HTS resides in skin dryness and an insufficient water content of the epidermis [9]. Dehydrated scar conditions are the main cause of pruritus, which is not only considered a sensation disorder but a relevant clinical parameter. In fact, itchy and dry scars are closely related to structural deficits of the typical scar tissue. Fibrous tissue tends to have less skin appendages such as sebaceous and sweat glands and thus produces less moisturizing secretions, which leads to skin dryness, less flexibility, and a rigid scar texture. Moreover, a thin epidermis with parallelly orientated collagen fibers can impair the epidermal barrier function, which is marked by transepidermal water loss (TEWL). Severe and widespread burn scars, for example, hold the risk of an incomplete reepithelialization of the epidermis due to a lack of stem cell reservoirs. It happens frequently that particularly those skin layers such as the basal layer of the epidermis are impaired by deeper and widespread damage of the skin. However, these components of the skin are able to provide stem cells that differentiate into functional skin or epithelial cells. Thus, a less functional epidermis enhances transepidermal water loss and a reduction of moisture. Therefore, patients suffer from dry, itchy, and painful scars followed by reddened and vulnerable skin. When protective features of epidermal layers are deficient, the skin reacts more sensitively to external factors and is more accessible for various pathogens, which can lead to progressive infections. In this context, medical needling ensures epidermal integrity in terms of regulating TEWL and reducing the risk of dehydration.
Measurements with the Corneometer and Tewameter have shown that PCI improves the moisture content of the skin, which is closely related to the regulation of TEWL. Various studies on the effect of medical needling on moisture revealed remarkable improvements regarding skin roughness and rigidity, which both depend on the water content of the skin (- Fig. 34.9).

Conservative treatments for scars with deficits in moisture and TEWL include silicone patches or diverse moisturizing creams that are established cosmetic therapy methods. Provided that they are used permanently and several times a day over a longer period of time, they can improve the scar condition to a temporary extent. Concerning the water content of the skin, the fluid silicone gel preserves the moisture content, but does not affect the skin's own potential to produce sufficient moisture [10]. A permanent application of oily creams keeps the skin moist and smooth, but does not improve the scar in quality by itself. TEWL is least affected, as the epidermal barrier function is not influenced by the silicone. Once again, short-term results are frequent and considering TEWL, there are less satisfying results. These kinds of methods have a preventative character and are rather suited for short-term solutions. In the case of HTS or keloids nonablative, conventional treatments can be used in addition to needling or other invasive therapy methods as complementary therapies. Ablative treatments achieve exactly the inverted effect in terms of destroying epidermal structures and holding the risk of degradation. On the contrary, the established method of medical needling preserves the epidermis and improves the epidermal barrier function. The significant impact on the skin's water balance is manifested by regulating moisturizing processes and the passive movement of water (TEWL) through the epidermis.
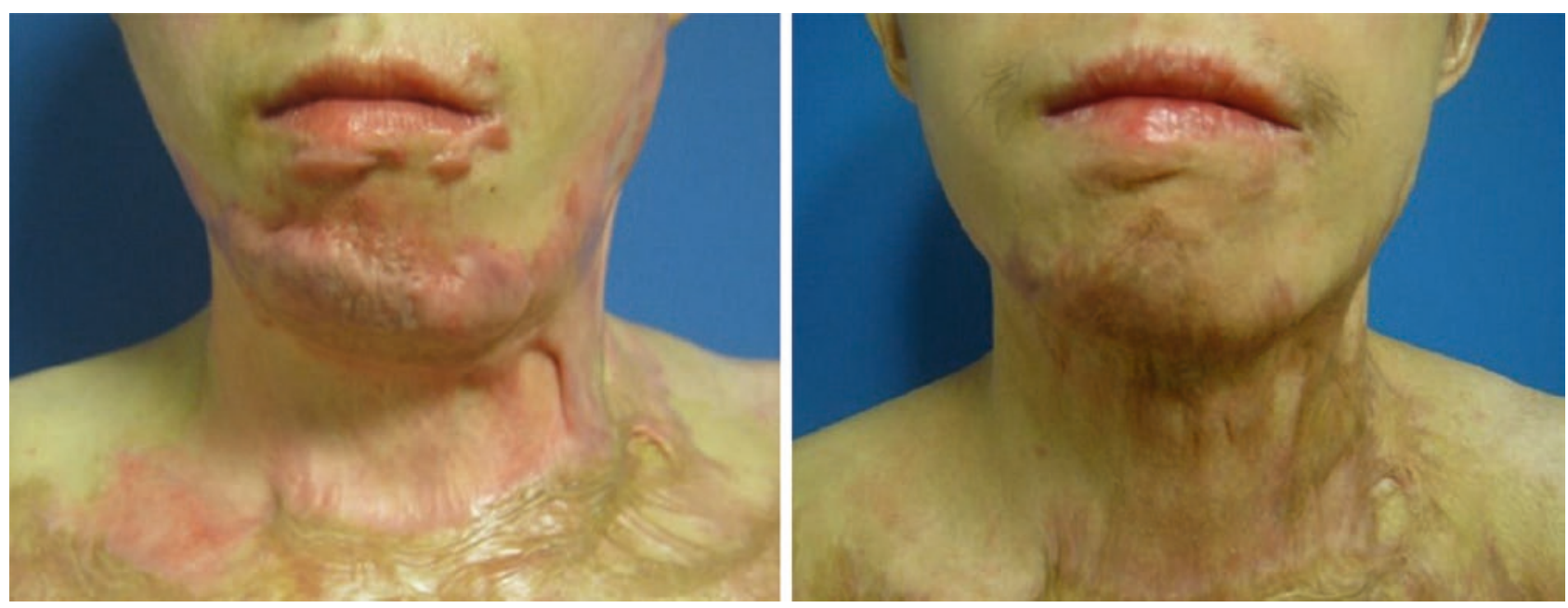

- Fig. 34.9 Patient 3, frontal shot, preoperatively (left) and one year postoperatively after needling (right). Areas treated include the face, perilabial, chin, and neck. Reduced erythema and greatness of the scar. Less tension in the fibrotic area 


\subsection{Conclusion}

Based on the diverse profile of medical needling regarding different parameters of HTS and keloids, this therapy method is not only an innovative approach, but also rather an effective treatment method of problematic scars. The aim of improving the scar in quality and function is realized by a modified wound-healing cascade, which activates relevant signal transduction pathways for an effective regeneration. After needling, dysfunctional scar tissue gains vital features of healthy skin and shows functional progression months and years after needling. The focus is clearly set on sustainability, which is the major challenge for an effective treatment of HTS and keloids. As underlying causes for the development of these types of scars are various and the clinical research potential on HTS and keloids is still not yet exhausted, medical solutions are required from modern medicine. Common therapy approaches are characterized by different moderate success that does not provide long-lasting outcome. Improvements are temporarily restricted and rather accumulate in the beginning of the therapy. HTS and keloids often tend to reach a nonresponder status in the course of repetitive conventional treatments. Surgical interventions are less attractive for the affected people and are often followed by a structural degradation up to uncontrolled cell death. In terms of not confronting the patients with serious consequences and other complications, medical treatment should be scientifically defined as effective and sustainable. PCI comes very close to what is expected from a medical point of view not only for the supplier side but especially for the demand side. Innovative and patient-friendly therapy approaches aim to be more convenient for patients relative to the required effort. In this context, medical needling seems to be a very promising therapy method that guarantees persistent effect on HTS and keloids. PCI displays a relatively simple and controlled treatment of difficult scars that opens new pathways for modern medicine.

\section{Take-Home Messages}

- HTS and keloids are pathological scars with a characteristic histological and clinical profile.

- Persistent lesions to deeper skin layers promote the development of HTS and keloids followed by an inflammatory response.

- Medical approach and success have been quite restricted to minimal outcomes and improvement in the past.
- The greater knowledge of pathomechanisms allows the establishment of targeted intervention.

- Medical needling overcomes deficits of conventional treatment therapies combining minimal invasivity and long-lasting effects at the same time.

- Important clinical endpoints (erythema, pruritus, pain, and moisture) and not only clinical parameters are positively affected by PCI.

- Dysfunctional scar tissue gains vital and healthy features after treatment with PCI.

- Outcomes are up-to-date and underlined by evidence-based clinical research.

\section{References}

1. Aust MC, Reimers K, Kaplan HM, et al. Percutaneous collagen induction-regeneration in place of cicatrisation? J Plast Reconstr Aesthet Surg. 2011;64:97-107. https://doi.org/10.1016/j. bjps.2010.03.038.

2. Aust MC, Fernandes D, Kolokythas P, Kaplan HM, Vogt PM. Percutaneous collagen induction therapy: an alternative treatment for scars, wrinkles and skin laxity. Plast Reconstr Surg. 2008;121(4):1421-9. https://doi.org/10.1097/01.prs.0000304612. 72899.02 .

3. Aust MC, Reimers K, Repenning C, et al. Percutaneous collagen induction: minimally invasive skin rejuvenation without risk of hyperpigmentation - fact or fiction? Plast Reconstr Surg. 2008;122:1553-63. https://doi.org/10.1097/PRS.0b013e318188 $245 \mathrm{e}$.

4. Aust MC, Bathe S, Fernandes D. Illustrated guide to percutaneous collagen induction. 1st ed. Berlin: KVM; 2013.

5. Aust MC, Knobloch K, Reimers K, Redeker J, Ipaktchi R, Altintas MA, Gohritz A, et al. Percutaneous collagen induction therapy: an alternative treatment for burn scars. Burns. 2010;36:836-43. https://doi.org/10.1016/j.burns.2009.11.014.

6. Laws RA, Finley EM, McCollough ML, Grabski WJ. Alabaster skin after carbon dioxide laser resurfacing with histologic correlation. Dermatol Surg. 1998;24:633-6. https://www.ncbi.nlm. nih.gov/pubmed/9648570. Accessed Jun 1998.

7. Fernandes D. Percutaneous collagen induction: an alternative to laser resurfacing. Aesthetic Surg J. 2002;22:307-9. https://doi. org/10.1067/maj.2002.126195.

8. Mustoe TA, Cooter RD, Gold MH, Hobbs FD, Ramelet AA, Shakespeare PG, Stella M, et al. International clinical recommendations on scar management. Plast Reconstr Surg. 2002;110:560 71. https://www.ncbi.nlm.nih.gov/pubmed/12142678. Accessed Aug 2002.

9. Anthonissen M, Daly D, Peeters R, Van Brussel M, Fieuws S, Moortgat P, Flour M, et al. Reliability of repeated measurements on post-burn scars with corneometer CM $825((\mathrm{R}))$. Skin Res Technol. 2015;21:302-12. https://doi.org/10.1111/srt.12193.

10. Bernstein LJ, Kauvar AN, Grossman MC, Geronemus RG. The short- and long-term side effects of carbon dioxide laser resurfacing. Dermatol Surg. 1997;23:519-25. https://www.ncbi.nlm. nih.gov/pubmed/9236869. Accessed Jul 1997. 
Open Access This chapter is licensed under the terms of the Creative Commons Attribution 4.0 International License (http://creativecommons. $\mathrm{org} /$ licenses/by/4.0/), which permits use, sharing, adaptation, distribution and reproduction in any medium or format, as long as you give appropriate credit to the original author(s) and the source, provide a link to the Creative Commons license and indicate if changes were made.

The images or other third party material in this chapter are included in the chapter's Creative Commons license, unless indicated otherwise in a credit line to the material. If material is not included in the chapter's Creative Commons license and your intended use is not permitted by statutory regulation or exceeds the permitted use, you will need to obtain permission directly from the copyright holder. 